Pathologe 2011 · 32:360-361

DOI 10.1007/s00292-011-1441-3

Online publiziert: 18. August 2011

(c) Springer-Verlag 2011
H.H. Kreipe

Institut für Pathologie, Medizinische Hochschule Hannover

\section{Pathologie der Infektionskrankheiten}

Nur bei einem kleineren Teil der pathologisch-anatomischen Befunde ist dem mikroskopischen Diagnostiker eine eindeutige ätiologische Zuordnung möglich. Über die Deskription des histologischen Erscheinungsbilds hinaus eine Erkrankung auf ihre Ursachen zurückführen und sie daran zweifelsfrei identifizieren zu können, gehört zu den besonders befriedigenden Seiten der pathologischen Diagnostik. Dies trifft insbesondere für die infektiösen Erkrankungen zu, auch wenn die Erregersuche häufig mühsam und zeitaufwendig ist. Mit immunhistochemischen und molekularpathologischen Verfahren stehen allerdings mittlerweile unterstützende Techniken zur Verfügung, die die Suche vereinfachen und präzisieren.

Nahezu alle Darstellungen der speziellen Organpathologie enthalten auch ein Infektionskapitel, sodass nicht alle relevanten Infektionserkrankungen in dem vorliegenden Themenheft von Der Pathologe, das notwendigerweise eine Auswahl darstellen muss, repräsentiert sind. Einige geplante Übersichten konnten nicht rechtzeitig von den Autoren fertiggestellt werden, sodass sie trotz ihrer Bedeutung nicht mehr in dieses Themenheft gelangten. Betroffen sind die Themen Helminthosen, infektiöse Enzephalitiden und die Helicobacter-Infektion. Da die granulomatösen Entzündungen schon in anderen Themenheften gesondert gewürdigt wurden, kommen sie hier nur als Unterthema vor.

Eine infektiöse Erkrankung, bei der der entscheidende Hinweis zumeist aus der pathologischen Diagnostik stammt, ist der M. Whipple. Nicht selten haben die Patienten eine diagnostische Odyssee und eine Vielzahl von Arztkontakten hinter sich, sodass es trotz der relativ geringen Inzidenz des M. Whipple von großer Bedeutung ist, diese Differenzialdiagnose bei Biopsien aus verschiedenen Organgebieten, nicht nur dem Magen-DarmTrakt, in Betracht zu ziehen. Das Zusammenspiel von klinischer und pathologischer Diagnostik bei dieser überaus komplexen Infektionserkrankung wird in einem Beitrag aus Berlin geschildert.

$\mathrm{Zu}$ den häufigsten Biopsien im Routinebetrieb eines Instituts für $\mathrm{Pa}$ thologie gehören die Kolonschleimhautpräparate. Im Gegensatz zum M. Whipple gelingt hier eine ätiologische Zuordnung nicht immer, wenngleich eine Infektion die wichtigste Differenzialdiagnose bei vielen entzündlichen Kolonerkrankungen darstellt. Diese Thematik wird in 2 Beiträgen aus Dresden aufgegriffen.

Nahezu dasselbe lässt sich über das Integument sagen, das als Grenzflächenorgan sehr häufig von infektiös bedingten Entzündungen betroffen ist. Der Erregernachweis stellt hier die entscheidenden Weichen für die Therapie, und gar nicht selten gelingt dieser rasch und eindeutig im Rahmen einer pathologisch-anatomischen Untersuchung von Hautbiopsien. Hierauf wird in einem Beitrag aus der dermatologischen Klinik der Medizinischen Hochschule Hannover (MHH) eingegangen.

An der Schnittstelle zwischen Infektions- und Tumorpathologie steht eine Reihe von Erregern. Das Epstein-Barr-Virus als Ursache der Posttransplantations- lymphoproliferation (PTLD) wurde bereits in dem vorangegangenen Themenschwerpunkt „Transplantationspathologie " in Der Pathologe beschrieben. In diesem Schwerpunktheft wird mit dem humanen Papillomavirus ein weiteres onkogenes humanes Virus vorgestellt, und in dem Beitrag aus München wird die $\mathrm{Pa}$ thogenese der viralen Tumorinduktion besonders gewürdigt.

Eine mögliche onkogene Rolle beim Menschen wird auch dem Polyomavirus zugesprochen, wobei sich, abgesehen vom Merkel-Zell-Karzinom, widersprüchliche Angaben in der Literatur finden. Von unbestrittener Bedeutung sind Polyomaviren jedoch für die Transplantationspathologie und hier insbesondere die Nephropathologie, was in einer Übersicht aus Hannover thematisiert wird.

Organtransplantierte Patienten sind nicht nur wegen der notwendigen Immunsuppression einem erhöhten Infektionsrisiko ausgesetzt, sondern sie können auch durch die Transplantation eines fremden Organs selbst gegenüber Krankheitserregern exponiert werden. Prätransplantationsdiagnostik und auch die Autopsie von Organspendern sind hier von besonderer Bedeutung. Für die histopathologische Sofortuntersuchung von Spenderorganen und Spenderbefunden stehen mittlerweile für die 7 Regionen der Deutschen Stiftung Organtransplantation jeweils 1 bis 2 Institute in ständiger Rufbereitschaft zur Verfügung. Nicht immer gelingt es, die entscheidende Diagnose rechtzeitig zu stellen. Solch ein Fall wird in einem Beitrag aus Bielefeld in Zusammenarbeit mit der Tierärztlichen Hoch- 
schule Hannover vorgestellt. Alle bis auf einen der 5 Organempfänger, die Organe von einer Rabies infizierten Spenderin erhalten hatten, erkrankten. Lediglich ein Förster, der noch aktiven Impfschutz be$\mathrm{saß}$, blieb verschont.

Eine andere Form der Immundefizienz, die mit einem besonderen Spektrum an Infektionserkrankungen verbunden ist, wird durch die „Human-immunodeficiency-virus“(HIV)-Infektion bzw. das erworbene Immundefektsyndrom repräsentiert. Das Spektrum der HIV-assoziierten pulmonalen Infektionen wird in einer gesonderten Übersicht zusammengefasst.

Die Zytomegalievirusinfektion stellt eine oft anzutreffende opportunistische Infektion dar, in dem sie gehäuft bei Immunkompromittierten vorkommt. Hier verursacht sie v. a. gastrointestinale und pulmonale Symptome. Aber auch bei Immungesunden treten Infektionen mit diesem humanen Herpesvirus auf. Dieser Virusinfektion ist ein eigener Beitrag gewidmet, der aus dem Institut für Pathologie der Universität Köln stammt.

Von herausragender Bedeutung sind die infektiös bedingten Lymphadenitiden, die klinisch und gelegentlich auch histopathologisch neoplastische Lymphoproliferationen imitieren können. Die Kenntnis der verschiedenen Reaktionsformen des lymphatischen Gewebes ist hier von Bedeutung, um eine sichere Abgrenzung von malignen Lymphomen herbeizuführen. Auch ohne Erregernachweis erlaubt darüber hinaus das Muster der Reaktion häufig Rückschlüsse auf die Ätiologie bzw. den zugrunde liegenden Erreger.

Jedem, der mit Medizin in Entwicklungsländern in Berührung kommt, wird schnell deutlich, welche überragende Bedeutung infektiologische Fragestellungen dort besitzen, die auch einen großen Anteil am pathologischen Untersuchungsgut ausmachen. Durch eine verbesserte Diagnostik in spezialisierten Instituten für Mikrobiologie und Virologie sowie effiziente Therapien haben die Infektionen in der täglichen pathologischen Diagnostik hierzulande an Stellenwert verloren und begegnen uns, abgesehen vom Helicobacter pylori zumeist nur noch in besonderen Situationen wie Immundefizienz oder Organtransplantation. Aber zuneh- mend treten eingeschleppte tropische Erreger in Erscheinung. In dem vorliegenden Themenheft werden hiesige Infektionen, an deren Diagnostik die Pathologie einen entscheidenden Anteil hat, vorgestellt. Einem weiteren Themenheft wird es vorbehalten bleiben, das histopathologische Erscheinungsbild von häufigen Tropenerkrankungen abzuhandeln. Den $\mathrm{Au}-$ toren dieser Ausgabe wird für ihre Bereitschaft, einen Beitrag beizusteuern, besonders gedankt.

Die Infektionspathologie kommt der kriminalistischen Seite unserer Tätigkeit besonders nah und hält nicht selten das besonders befriedigende Erfolgserlebnis bereit, einen komplizierten Fall mit einem Schlag gelöst zu haben. In diesem Sinn wünsche ich allen Leserinnen und Lesern dieser Ausgabe von Der Pathologe eine stimulierende Lektüre.

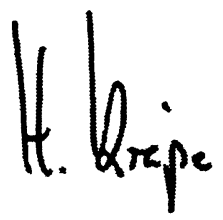

\section{H.H. Kreipe}

\section{Korrespondenzadresse}

Prof. Dr. H.H. Kreipe

Institut für Pathologie,

Medizinische Hochschule Hannover

Carl-Neuberg-Str. 1, 30625 Hannover

kreipe.hans@mh-hannover.de

\section{Onkologische Notfälle}

Ein onkologischer Notfall kann durch die Manifestation des malignen Grundleidens, durch Komplikationen der Therapie aber auch durch Störungen unabhängig von der Grunderkrankung hervorgerufen werden. In der Onkologie sind sogar präventive Interventionen möglich, da nahezu alle gefährlichen Situationen vorhersehbar sind

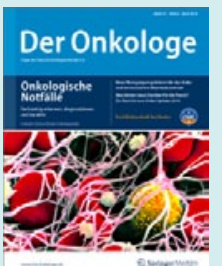

Unter dem Leitthema "Onkologische Notfälle" werden in der Ausgabe 04/2010 der Zeitschrift "Der Onkologe" die klassischen Themen wie z.B. urologische

Aspekte und chirurgische Möglichkeiten behandelt. Ein neuer Bereich der onkologischen Notfallmedizin ist die Pathologie, die frühzeitig die richtigen Weichen für eine Anschlusstherapie nach dem Notfall einleiten kann. Das Leitthemenheft beinhaltet unter anderem folgende Beiträge:

- Strahlentherapeutische Interventionen bei onkologischen Notfällen

- Notfälle in der Pathologie. Was kann der Pathologe leisten?

- Gastrointestinaler Notfall in der Onkologie

- Chirurgische Notfälle unter Chemo- und Strahlentherapie

Bestellen Sie diese Ausgabe zum Preis von EUR 34,- zzgl. Versandkosten bei:

Springer Customer Service Center GmbH Kundenservice Zeitschriften

Haberstr. 7

69126 Heidelberg

Tel.: +49 6221-345-4303

Fax: +49 6221-345-4229

leserservice@springer.com

www.deronkologe.de

P.S. Vieles mehr rund um Ihr Fachgebiet finden Sie auf www.springermedizin.de 